\title{
Compte-rendu de Owen Bennett, The Brexit Club: The Inside Story of the Leave Campaign's Shock Victory
}

\section{Pauline Schnapper}

\section{OpenEdition}

\section{Journals}

Édition électronique

URL : http://journals.openedition.org/rfcb/1258

DOI : $10.4000 /$ rfcb. 1258

ISSN : 2429-4373

Éditeur

CRECIB - Centre de recherche et d'études en civilisation britannique

Référence électronique

Pauline Schnapper, «Compte-rendu de Owen Bennett, The Brexit Club: The Inside Story of the Leave Campaign's Shock Victory», Revue Française de Civilisation Britannique [En ligne], XXII-2 | 2017, mis en ligne le 30 mai 2017, consulté le 27 avril 2019. URL : http://journals.openedition.org/rfcb/1258 ; DOI $10.4000 /$ rfcb. 1258

Ce document a été généré automatiquement le 27 avril 2019

\section{c) $(9)$}

Revue française de civilisation britannique est mis à disposition selon les termes de la licence Creative Commons Attribution - Pas d'Utilisation Commerciale - Pas de Modification 4.0 International. 


\title{
Compte-rendu de Owen Bennett, The Brexit Club: The Inside Story of the Leave Campaign's Shock Victory
}

\author{
Pauline Schnapper
}

\section{RÉFÉRENCE}

Owen Bennett, The Brexit Club: The Inside Story of the Leave Campaign's Shock Victory, Londres, Biteback Publishing, 2016, ISBN: 978-1-78590-098-3, 340 pages.

1 L'ouvrage d'Owen Bennett, journaliste au Huffington Post, est en quelque sorte le pendant de celui de Craig Oliver sur la campagne du Remain. Il s'agit ici de retracer au jour le jour la façon dont s'est déroulée la campagne du Leave en suivant ses principaux acteurs depuis les élections législatives de mai 2015 jusqu'au lendemain du référendum. Contrairement à Oliver, qui en était une des figures centrales, l'approche ici est extérieure, "objective", et cherche moins à expliquer qu'à décrire, ce qui fait l'intérêt mais aussi les limites de l'exercice chez l'auteur, qu'on peut imaginer soumis à la pression de son éditeur pour publier au plus vite.

Bennett fait clairement apparaître comment les partisans du Brexit ont commencé à s'organiser très tôt, dès juin 2015, à une époque où Cameron entretenait la fiction que son choix de rester ou pas dans l'Union européenne dépendrait uniquement du résultat de la renégociation qu'il allait entreprendre à Bruxelles. Le noyau dur idéologique de la campagne, autour de Daniel Hannan, Matthew Elliott, Bernard Jenkin ou John Baron côté conservateur, Kate Hoey, Graham Stringer ou John Mills côté travailliste, était déjà bien en place à cette époque, sans compter bien sûr les rangs du UKIP.

3 Ce qui ressort ensuite de cette description, c'est que la campagne a été marquée, autant peut-être que celle de ses adversaires, par des divisions et des tensions parfois fortes entre des campagnes et des personnalités opposées. On sait que deux organisations rivales ont tenté d'être reconnues par la Electoral Commission comme l'organisation 
officielle qui pourrait recevoir les fonds publics prévue pour la campagne: Vote Leave, dirigée par Matthew Elliott et Dominic Cummings et regroupant une majorité de Conservateurs et quelques travaillistes; Leave.EU, financée par le milliardaire Arron Banks et dominée par Nigel Farage et les cadres du UKIP, sauf leur seul député Douglas Carswell qui était membre de Vote Leave. Il s'est ajouté à cette organisation duale un troisième groupe, Grassroots Out, créé en décembre 2015 et qui s'est appuyé, comme son nom l'indique, sur une organisation de terrain et a essayé de faire le lien entre les deux autres. Bennett raconte longuement les tiraillements, à la fois personnels et sur la tactique à adopter, entre ces différents acteurs tout au long de la campagne. Les relations étaient si mauvaises qu'en décembre 2015, Banks soupçonna Vote Leave d'avoir recruté des détectives privés pour le surveiller (p. 150)...

4 Avec une campagne aussi chaotique, on peut se demander comment le vote pour le Brexit l'a emporté. L'auteur ne répond pas à cette question, mais deux hypothèses peuvent être avancées. Premièrement, il se pourrait que la campagne n'ait pas eu beaucoup d'effet sur le choix des électeurs, déjà décidés (dans un sens ou dans un autre, d'ailleurs) avant février 2016. La deuxième, c'est que malgré les ratés de la campagne, ses organisateurs ont su intelligemment s'adapter à ce que leur disaient les sondages et autres focus groups. Se sachant en position de faiblesse sur les arguments économiques, les animateurs du Leave ont réussi à recentrer le débat sur la question sur laquelle ils se savaient en bien meilleure position, l'immigration. Même les Conservateurs favorables à l'immigration, comme Hannan, se rallièrent sans peine à cette stratégie qui était manifestement si efficace. Ils se démarquèrent tout de même de l'affiche rendue publique par Nigel Farage le jour même de l'assassinat de la députée Jo Cox, qui montrait une file de réfugiés sous le titre "Breaking Point - the EU has failed us all", qui selon l'auteur choqua la plupart des ténors du camp du Leave.

5 Plus généralement, les Brexiteers ont réussi, après un début moins convaincant, à imposer les thèmes de la campagne et à mettre le camp du Remain sur la défensive grâce au slogan fourre-tout "Take Back Control" et à l'insistance sur les sommes qui seraient économisées en sortant de l'UE et qui pourraient être réinvesties dans le NHS. Les Brexiters, qui savaient qu'ils devaient recueillir les voix d'au moins une partie des électeurs travaillistes, ont aussi bénéficié de la faiblesse de la campagne pro-europénne du parti travailliste et de son leader, inaudible pendant la campagne, et de l'apport de personnalités populaires comme Boris Johnson à partir de février.

Bennett montre aussi que l'opposition parfois affichée entre une organisation "respectable", Vote Leave, qui aurait utilisé le thème de la souveraineté, et une autre, Leave.EU, qui n'aurait pas lésiné sur les mensonges et attisé les haines anti-immigrants, était simpliste. Vote Leave n'a en réalité pas hésité à recourir à des méthodes plus que discutables, comme lorsque des jeunes militants se sont introduits dans les locaux de la CBI pour perturber un discours de David Cameron ou lorsque l'organisation a publié une liste de criminels européens présents au Royaume-Uni (p. 236). En ce sens les deux campagnes ont bien été complémentaires et se sont renforcées l'une l'autre plutôt qu'affaiblies.

7 Cet ouvrage bien informé sur les coulisses de la campagne du Leave et fourmillant d'anecdotes plus ou moins intéressantes donne une bonne idée de l'atmosphère qui régnait dans la campagne jusqu'à la victoire finale du 23 juin. Il ne faut pas en attendre davantage que ce qu'il annonce lui-même, c'est-à-dire un récit au jour le jour des petites tactiques et des relations compliquées entre ses protagonistes. On ne peut s'empêcher de 
rester un peu sur sa faim si l'on y cherche une analyse approfondie des raisons du vote pour le Brexit.

\section{AUTEURS}

\section{PAULINE SCHNAPPER}

Université Paris 3 